\title{
Do GDPs explain the risks of GA before referring?
}

\author{
Appropriate consent and referral for general anaesthesia - a survey in the Paediatric Day Care Unit, Barnsley DGH \\ NHS Trust, South Yorkshire A. M. Patel Br Dent J 2003; 196: 275-277
}

\section{Background}

In November 1998 the General Dental Council introduced guidelines for dental practitioners when referring a patient for general anaesthesia (GA). The practitioner is required to explain the risks associated with GA and the alternatives, give a detailed medical history and a clear justification for providing GA in the letter of referral.

\section{Method}

A survey was administered on 202 parents or guardians, which aimed to investigate whether they felt that their dental practitioners had advised them of any risks of GA prior to referral. A record was also made if any reasons were given for the provision of GA in the letter of referral.

\section{Results}

The majority of the parents or guardians (66\%) felt that they were not informed of any of the risks of GA and 25\% felt that they were. From the letters of referral, 37\% contained a reason for GA and 63\% did not give any reason or justification for GA.

\section{Conclusion}

There is evidence that referring practitioners do not adequately explain the risks of the anaesthetic to parents or guardians of children undergoing GA. There is also a lack of clear justification in the letters of referral for providing GA.

\section{Practice implication}

It is essential that the alternatives and the risks of GA are discussed and if GA is still required, a clear justification should be contained in the letter of referral as part of informed consent. More importantly the referring practitioner should keep a contemporaneous record of this, preferably with a signature from the parent or guardian on agreement of referral.

\section{IN BRIEF}

- This paper highlights to the practitioner her/his duty of care and the need for valid informed consent.

- Points to consider prior to, and when referring patients for extractions under general anaesthesia.

- This paper also highlights to the practitioner the need to keep contemporaneous notes.

\section{COMMENT}

This timely paper closely follows a similar one also considering compliance with guidelines concerning referral of children for general anaesthesia (GA). ${ }^{1}$ The curtailment of GA in general dental practice and its transfer to hospital has stretched the ability of hospital oral surgery departments to meet the increased demand for the treatment of children under GA. The rationale for conducting this study is easy to understand. With the limited resources available in hospital, no child should receive a GA unless the indications for this are indisputable. Similarly the parents of the children referred should be informed about the morbidity and potential mortality of GA.

It is disturbing, therefore, to read in this paper that most parents questioned in this study were unaware of the risks of GA. It is possible that dentists find it hard to raise the emotive subject of mortality associated with GA when it is such a rare event.

Prior to the release of the guidelines, the ease of access to GA in general dental practice resulted in many children receiving GA. The spirit of the new regulations is not merely to transfer children to hospital where the risks associated with GA still exist. A child fatality associated with GA in hospital administered for simple dental treatment may still occur. It should therefore be gold standard practice to explore alternatives to GA prior to referral.

Another concern raised in this study is that a majority of referring dentists gave no reason or justification for referral for $\mathrm{GA}$. There is good evidence that sedation is a safe, acceptable and successful alternative to GA. ${ }^{2}$ Whereas in the USA an estimated $56 \%$ of dentists use nitrous oxide/oxygen sedation, there is still very limited availability of this technique in the UK especially within general dental practice.

The relevance of this paper is that it re-emphasises the responsibilities still incumbent on dentists when referring children to hospital for treatment under GA. I hope that this study will result in an increase in the availability of sedation services for children.

Alex Crawford. Hon. Clinical Lecturer,

Turner Dental Hospital and School, Manchester

1. Clayton M, Mackie I C. The development of referral guidelines for dentists referring children for extractions under general anaesthesia. Br Dent J 2003; 194: 561-565.

2. Blain $\mathrm{K}_{1}$ Hill FJ. The use of inhalation sedation and local anaesthetic as an alternative to GA for extractions in children. Br DentJ 1998; 184: 608-611. 